\title{
FACTORIZATION OF MEASURES AND NORMAL CONDITIONAL DISTRIBUTIONS
}

\author{
A. MAITRA AND S. RAMAKRISHNAN \\ (Communicated by William D. Sudderth)
}

\begin{abstract}
Let $(Y, \mathscr{C}, Q)$ be a probability space. If every probability measure $R$ on $\mathscr{B}^{1} \otimes \mathscr{C}$ with marginal $Q$ on $Y$ admits a factorization, where $\mathscr{B}^{1}$ is the Borel $\sigma$-field on the real line, $Q$ must be perfect. Conversely if $Q$ is perfect and $\mathscr{C}$ is $\aleph_{1}$-generated, then (a) for any measure $R$ on $\mathscr{A} \otimes \mathscr{C}$ with marginal $Q$, where $\mathscr{A}$ is any $\sigma$-field of subsets of a set $X$, there is a factorization; (b) for every tail-like sub- $\sigma$-field $\mathscr{D}$ of $\mathscr{C}$, there is a normal conditional distribution given $\mathscr{D}$. In special cases of interest, normal conditional distributions, satisfying additional desirable properties, are shown to exist.
\end{abstract}

1. Introduction. For a Bayesian statistician, whose inferences are based on the posterior distribution of the parameter given the sample space, the problem of factoring a measure on the product of the sample space and the parameter space is an important one. If $(Y, \mathscr{C})$ denotes the parameter space, $Q$ the prior distribution and $(X, \mathscr{A})$ the sample space, it is of interest to investigate conditions under which a measure $R$ on the product space with marginal $Q$ on $Y$ admits a factorization. A somewhat related problem is the problem of obtaining, on a probability space, conditional distributions given a sub- $\sigma$-field. The study of existence of proper, regular conditional distributions has been carried out in a number of papers, most notably [4, 2 and 5]. Since proper, regular conditional distributions need not exist for classically interesting sub- $\sigma$-fields in a standard Borel space, a weaker notion of conditional distribution, called normal conditional distribution, has been proposed in Dubins [5] and Blackwell and Dubins [2]. For a normal conditional distribution, conditional probabilities need only be finitely additive. The study of existence of normal conditional distributions, equivalent to that of measurable disintegrations as defined in [7 and 8], has since been of interest. It has been shown in Dubins and Heath [8] that for Luzin spaces, normal conditional distributions given many interesting sub- $\sigma$-fields, called tail-like $\sigma$-fields, do exist (whereas proper, regular conditional distributions need not). Statistical models and procedures based on finitely additive probabilities have been investigated (see, for example, Heath and Sudderth [9] and Lane and Sudderth [10]).

We study, in this paper, existence of factorizations that are finitely additive, for probability measures on a product space and also the existence of normal conditional distributions. $\S 2$ contains definitions and preliminaries. $\S 3$ contains results

Received by the editors May 18, 1987.

1980 Mathematics Subject Classification (1985 Revision). Primary 60A10, 60A05, 28A50; Secondary 28D05, 60G09.

Key words and phrases. Factorization, disintegration, conditional distribution, normal conditional distribution, perfect probability space, tail-like $\sigma$-field, measure-preserving transformation, invariant $\sigma$-field, symmetric $\sigma$-field. 
on factorization. The results in [ $\mathbf{1}$ and $\mathbf{3}$ ] are special cases of our Theorems 1 and 2. In $\S 4$ we use our results on factorizations to obtain a generalization (Theorem 4) of the Theorem of Dubins and Heath in [8], to perfect probability spaces with an $\aleph_{1}$-generated $\sigma$-field. Theorems 4 and 5 show that for certain classically interesting sub- $\sigma$-fields, the invariant $\sigma$-field in a probability space with a measure-preserving transformation, and the symmetric $\sigma$-field in a countably infinite product space, normal conditional distributions with further desirable properties exist.

2. Definitions and preliminaries. Throughout this paper, by a charge $\mu$ we shall mean a finitely additive, nonnegative function on a field of subsets of a set $X$ such that $\mu(X)=1$. By a measure we shall refer to a countably additive charge. Suppose $\mu$ is a charge on a field $\mathscr{F}$ of subsets of a set $X$ and that $\mathscr{G}$ is a subfield of $\mathscr{F}$. A subset $A$ of $X$ will be called $(\mu, \mathscr{G})$-thick if $\mu(B)=1$ for all $B \in \mathscr{G}$ such that $A \subseteq B$. For a subset $A$ of $X, \mathscr{F} \cap A$ will denote the collection $\{F \cap A: F \in \mathscr{F}\}$. Plainly $\mathscr{F} \cap A$ is a field or a $\sigma$-field according as $\mathscr{F}$ is a field or a $\sigma$-field. If $\mu$ is a charge (measure) on a field ( $\sigma$-field) $\mathscr{F}$ of subsets of $X$ and $A$ is $(\mu, \mathscr{F})$-thick, by the restriction $\nu$ of $\mu$ to $\mathscr{F} \cap A$, we mean the charge (measure) on $\mathscr{F} \cap A$ defined by $\nu(F \cap A)=\mu(F), F \in \mathscr{F}$. Conversely for any $A \subseteq X$, a field ( $\sigma$-field) $\mathscr{F}$ on $X$ and a charge (measure) $\nu$ on the field ( $\sigma$-field) $\mathscr{F} \cap A$, by the extension $\mu$ of $\nu$ to $\mathscr{F}$ we shall mean the charge (measure) $\mu$ on $\mathscr{F}$ defined by $\mu(F)=\nu(F \cap A), F \in \mathscr{F}$. Clearly $A$ is $(\mu, \mathscr{F})$-thick,.

A measure space $(Y, \mathscr{C}, Q)$ is perfect (or $Q$ is perfect) if for any realvalued $\mathscr{C}$ measurable function $f$, there is a Borel subset $B$ of the real line such that $B \subseteq f(Y)$ and $Q\left(f^{-1}(B)\right)=1$.

We shall need to use the following theorem of Sazonov [17].

THEOREM (SAZONOV). Let $(X, \mathscr{B}, \mu)$ be a perfect measure space and let $Y$ be $a(\mu, \mathscr{B})$-thick subset of $X$. Then the restriction of $\mu$ to $\mathscr{B} \cap Y$ is perfect if and only if for every countably generated sub- $\sigma$-field $\mathscr{A}$ of $\mathscr{B}$, there exists a $\mu$-null set $N_{\mathscr{A}} \in \mathscr{A}$ such that $E \cap Y \neq \varnothing$ for every $\mathscr{A}$-atom $E \subseteq X \backslash N_{\mathscr{A}}$.

The statement and proof of the theorem can also be found in [13].

Suppose $(Y, \mathscr{C}, Q)$ is a measure space such that $\mathscr{C}$ is $\lambda$-generated, where $\lambda$ is a cardinal. Let $C_{\xi}, \xi<\lambda$, be subsets of $Y$ such that $\mathscr{C}=\sigma\left(\left\{C_{\xi}, \xi<\lambda\right\}\right)$, where we have identified the cardinal $\lambda$ with its initial ordinal. Let $\phi$ denote the function on $Y$ into $\{0,1\}^{\lambda}$ defined by $\phi(y)(\xi)=I_{C_{\xi}}(y), \xi<\lambda$, where $I_{E}$ denotes the characteristic function of the set $E$. If $\mathscr{B}$ denotes the product of discrete $\sigma$-fields on $\{0,1\}^{\lambda}$, it is easy to verify that $\phi$ sets up an isomorphism between the $\sigma$-fields $\mathscr{C}$ and $\mathscr{B} \cap \phi(Y)$. Let $\widetilde{Q}$ be the extension of the measure $Q \phi^{-1}$ on $\mathscr{B} \cap \dot{\varphi}(Y)$ to $\mathscr{B}$. Clearly $\phi(Y)$ is $(\widetilde{Q}, \mathscr{B})$-thick. It is well known that equipped with the product of discrete topologies, $\{0,1\}^{\lambda}$ is a compact, Hausdorff space and $\mathscr{B}$ coincides with its Baire $\sigma$-field. Moreover the measure space $\left(\{0,1\}^{\lambda}, \mathscr{B}, \widetilde{Q}\right)$ is a compact (hence perfect) measure space (for the definition and properties of a compact measure space, see [11 and 16]). Thus the measure space $(Y, \mathscr{C}, Q)$ can be embedded isomorphically into the compact (hence perfect) measure space $\left(\{0,1\}^{\lambda}, \mathscr{B}, \widetilde{Q}\right)$ such that $Y$ is a $(\widetilde{Q}, \mathscr{B})$-thick set.

Let $(X, \mathscr{A})$ and $(Y, \mathscr{C})$ be measurable spaces. The product of the $\sigma$-fields $\mathscr{A}$ and $\mathscr{C}$ is denoted by $\mathscr{A} \otimes \mathscr{C}$. If $E \subseteq X \times Y$ and $x \in X, E_{x}$ will denote the set 
$\{y \in Y:(x, y) \in E\}$. We denote by $\pi_{X}\left(\pi_{Y}\right)$ the projection function from $X \times Y$ to $X(Y)$.

DEFINITION. Let $R$ be a measure on $(X \times Y, \mathscr{A} \otimes \mathscr{C})$. We say that $\left\{T_{x}\right\}$ is a factorization of $R$ if

(i) for each $x \in X, T_{x}$ is a charge on $\mathscr{C}$

(ii) for each $E \in \mathscr{A} \otimes \mathscr{C}$,

(a) the map $x \rightarrow T_{x}\left(E_{x}\right)$ is $\overline{\mathscr{A}}$-measurable, where $\overline{\mathscr{A}}$ is the completion of $\mathscr{A}$ w.r.t. $P=R \pi_{X}^{-1}$, the marginal of $R$ on $X$, and,

(b) $R(E)=\int T_{x}\left(E_{x}\right) d P(x)$.

For a measure $R$ on $(X \times Y, \mathscr{A} \otimes \mathscr{C})$, Pachl [14] has introduced the notion of disintegration of $R$, similar to our notion of factorization, the main difference being that for each $x, T_{x}$ is a measure on a sub- $\sigma$-field $\mathscr{C}_{x}$ of $\mathscr{C}$. Using routine measure-theoretic arguments and the well known result (see, for example [18]) that any charge on a field $\mathscr{C}^{\prime}$ can be extended as a charge on any field $\mathscr{C}$ containing $\mathscr{C}^{\prime}$, it can be shown that the existence of a disintegration of $R$ implies the existence of a factorization of $R$.

Let $(Y, \mathscr{C}, Q)$ be a measure space and let $\mathscr{D}$ be a sub- $\sigma$-field of $\mathscr{C}$. For $y \in Y$, the $\mathscr{D}$-atom containing $y$, to be denoted by $D(y)$, is the intersection of all sets in $\mathscr{D}$ containing $y$. Note that, in general $D(y)$ need not belong to $\mathscr{D}$.

The following definition of a normal conditional distribution is the same as in Blackwell and Dubins [2]. This is equivalent to the notion of measurable disintegration in [7 and 8].

DEFINITION. Say that a function $\mu$ on $Y \times \mathscr{C}$ into $[0,1]$ is a normal conditional distribution given $\mathscr{D}$ if

(i) for each $y \in Y, \mu(y, \cdot)$ is a charge on $\mathscr{C}$

(ii) for each $A \in \mathscr{C}, \mu(\cdot, A)$ is $\overline{\mathscr{D}}$-measurable, where $\overline{\mathscr{D}}$ is the completion of $\mathscr{D}$ with respect to $Q$

(iii) for $y_{1}, y_{2}$ in $Y, D\left(y_{1}\right)=D\left(y_{2}\right)$ implies $\mu\left(y_{1}, \cdot\right)=\mu\left(y_{2}, \cdot\right)$

(iv) for each $y \in Y, D(y)$ is $(\mu(y, \cdot), \mathscr{D})$-thick.

(v) for each $A \in \mathscr{C}$,

$$
Q(A)=\int \mu(y, A) d Q(y)
$$

We remark that because of (iv), (v) is equivalent to

$\left(\mathrm{v}^{\prime}\right)$ for each $A \in \mathscr{C}$ and $D \in \mathscr{D}$,

$$
Q(A \cap D)=\int_{D} \mu(y, A) d Q(y) .
$$

3. Factorization. Let $\mathscr{B}^{1}$ denote the Borel $\sigma$-field of the real line. Here are the two main theorems of this section.

THEOREM 1. Let $(Y, \mathscr{C}, Q)$ be a measure space. Suppose that for any measure $R$ on $\mathscr{B}^{1} \otimes \mathscr{C}$ with marginal $Q$ on $Y$, there exists a factorization of $R$. Then $Q$ is a perfect measure.

THEOREM 2. Suppose $(Y, \mathscr{C}, Q)$ is a perfect measure space and $\mathscr{C}$ is $\aleph_{1}$ generated. If $(X, \mathscr{A})$ is any measurable space and $R$ a measure on $\mathscr{A} \otimes \mathscr{C}$ with marginal $Q$ on $Y$, then there exists a factorization $\left\{T_{x}\right\}$ of $R$. Furthermore the factorization $\left\{T_{x}\right\}$ can be chosen so that for any countably generated sub- $\sigma$-field $\mathscr{D}$ 
of $\mathscr{C}$, there is a set $N \in \mathscr{A}$ with $P(N)=0$ such that for $x \notin N, T_{x}$ is a measure on $\mathscr{D}$. (Here $\aleph_{1}$ denotes the first uncountable cardinal and for any cardinal $\lambda$, we say that a $\sigma$-field $\mathscr{C}$ is $\lambda$-generated if there is a family $\mathscr{C}_{0}$ of sets such that $\operatorname{Card}\left(\mathscr{C}_{0}\right) \leq \lambda$ and $\mathscr{C}$ is the $\sigma$-field generated by $\mathscr{C}_{0}$.)

If $(Y, \mathscr{C}, Q)$ is a measure space, a necessary and sufficient condition for any measurable space $(X, \mathscr{A})$ and any measure $R$ on $(X \times Y, \mathscr{A} \otimes \mathscr{C})$ with marginal $Q$ on $Y$ to admit a disintegration is that $(Y, \mathscr{C}, Q)$ be a compact measure space. This theorem was proved by Pachl in [14]. Since every compact measure space is perfect, in view of our results, for a $\aleph_{1}$-generated $\sigma$-field, the difference between compactness and perfectness of a countably additive measure lies in the existence of disintegrations as opposed to factorizations. We mention in this connection that in [13], Musial has an example of a measure on a $\aleph_{1}$-generated $\sigma$-field which is perfect but not compact. Thus there are measure spaces for which factorizations exist but not disintegrations. Whether Theorem 2 can be extended to the case when $\mathscr{C}$ is not $\aleph_{1}$-generated is still open.

PROOF OF THEOREM 1 . Let $f$ be a realvalued $\mathscr{C}$-measurable function. We shall show that there is a Borel subset $B$ of the real line such that $B \subseteq f(Y)$ and $Q\left(f^{-1}(B)\right)=1$. Let $R$ be the measure on $\mathscr{B}^{1} \otimes \mathscr{C}$ defined by

$$
R(E)=\int I_{E^{y}}(f(y)) d Q(y), \quad \text { for } E \in \mathscr{B}^{1} \otimes \mathscr{C}
$$

where $E^{y}=\{x:(x, y) \in E\}$.

So, by hypothesis, there exists a factorization $\left\{T_{x}\right\}$ of $R$. Consider the set $G=\{(x, y): y \in Y$ and $f(y)=x\}$. It is easily seen that $G \in \mathscr{B}^{1} \otimes \mathscr{C}$ and $R(G)=1$.

So $\int T_{x}\left(G_{x}\right) d P(x)=1$, where $P=R \pi_{X}^{-1}=Q f^{-1}$. Also since $x \rightarrow T_{x}\left(G_{x}\right)$ is $\overline{\mathscr{B}}^{1}$-measurable, there is a Borel set $B$ such that $B \subseteq\left\{x: T_{x}\left(G_{x}\right)=1\right\}$ and $P(B)=1$, i.e. $Q\left(f^{-1}(B)\right)=1$. Clearly $B \subseteq f(Y)$ since for $x \notin f(Y), G_{x}$ is empty. This completes the proof.

The above proof, suggested to us by B. V. Rao, is simpler than our original proof.

We shall use the following lemma to prove Theorem 2. It is probably known, but since we cannot cite a reference, we include a proof for completeness.

LEMMA 1. Let $\lambda$ be an infinite cardinal. Suppose $(X, \mathscr{A})$ is a measurable space and $\mathscr{B}$ is the Baire $\sigma$-field on $\{0,1\}^{\lambda}$. Let $R$ be a measure on $\mathscr{A} \otimes \mathscr{B}$. Then, for each $x \in X$, there is a measure $T_{x}$ on $\mathscr{B}$ such that

(a) for each $B \in \mathscr{B}$, the map $x \rightarrow T_{x}(B)$ is $\overline{\mathscr{A}}$-measurable, where $\overline{\mathscr{A}}$ is the completion of $\mathscr{A}$ w.r.t. $P$, the marginal of $R$ on $X$, and,

(b) for each $E \in \mathscr{A} \otimes \mathscr{B}$,

$$
R(E)=\int T_{x}\left(E_{x}\right) d P(x)
$$

ProOF. By a lifting theorem of Maharam (see [12], for example), choose a linear lifting $l$ on $\mathscr{L}_{\infty}(X, \overline{\mathscr{A}}, P)$. Let $\mathscr{A}^{*}$ be the $\sigma$-field on $X \times\{0,1\}^{\lambda}$ of sets of the form $A \times\{0,1\}^{\lambda}$ with $A \in \mathscr{A}$. For each $f \in C\left(\{0,1\}^{\lambda}\right)$, the space of continuous functions on $\{0,1\}^{\lambda}$, let $f^{*}$ be the function defined by $f^{*}(x, y)=f(y)$, for all 
$(x, y) \in X \times\{0,1\}^{\lambda}$ and let $E_{R}\left(f^{*} \mid \mathscr{A}^{*}\right)$ denote the conditional expectation of $f^{*}$ with respect to $\mathscr{A}^{*}$ under the measure $R$. Plainly $E_{R}\left(f^{*} \mid \mathscr{A}^{*}\right)$ induces a unique element, to be denoted by $\overline{E_{R}\left(f^{*} \mid \mathscr{A}^{*}\right)}$ of $\mathscr{L}_{\infty}(X, \overline{\mathscr{A}}, P)$. Define

$$
l_{x}(f)=l\left(\overline{E_{R}\left(f^{*} \mid \mathscr{A}^{*}\right)}\right)(x), \quad x \in X, f \in C\left(\{0,1\}^{\lambda}\right) .
$$

It is easily verified that for each $x \in X, l_{x}$ is a normalized, positive, linear functional on $C\left(\{0,1\}^{\lambda}\right)$. By the Riesz representation theorem, there exists a unique measure $T_{x}$ on $\mathscr{B}$ such that $l_{x}(f)=\int f d T_{x}$ for $f \in C\left(\{0,1\}^{\lambda}\right)$. It is a routine verification that $T_{x}, x \in X$, satisfy the assertion of the lemma.

PROOF OF THEOREM 2. As stated earlier, since $\mathscr{C}$ is $\aleph_{1}$-generated, we may assume that $Y \subseteq\{0,1\}^{\omega_{1}}$ (where $\omega_{1}$ is the first uncountable ordinal), $\mathscr{C}=\mathscr{B} \cap Y$ where $\mathscr{B}$ is the Baire $\sigma$-field of $\{0,1\}^{\omega_{1}}, \widetilde{Q}$ is the extension of $Q$ from $\mathscr{B} \cap Y$ to $\mathscr{B}$ and $Y$ is a $(\widetilde{Q}, \mathscr{B})$-thick set. Also since $\mathscr{B}$ is $\aleph_{1}$-generated, we can and do fix countably generated sub- $\sigma$-fields $\mathscr{B}_{\xi}, 0 \leq \xi<\omega_{1}$, of $\mathscr{B}$ satisfying

(a) $\mathscr{B}_{0}=\left\{\phi,\{0,1\}^{\omega_{1}}\right\}$, and $\xi<\eta<\omega_{1}$ implies $\mathscr{B}_{\xi} \subseteq \mathscr{B}_{\eta}$,

(b) $\mathscr{B}_{\xi}=\sigma\left(\bigcup\left\{\mathscr{B}_{\eta}: \eta<\xi\right\}\right)$, if $\xi$ is a limit ordinal

(c) $\mathscr{B}=\bigcup\left\{\mathscr{B}_{\xi}: \xi<\omega_{1}\right\}$.

We set $\mathscr{B}_{\omega_{1}}=\mathscr{B}$.

Since $Q$ is perfect, it follows by Sazonov's theorem that for each $\xi<\omega_{1}$, there exists $M_{\xi} \in \mathscr{B}_{\xi}$ such that $\widetilde{Q}\left(M_{\xi}\right)=0$ and if $B$ is a $\mathscr{B}_{\xi}$-atom and $B \subseteq\{0,1\}^{\omega_{1}} \backslash M_{\xi}$, then $B \cap Y \neq \varnothing$. Set $N_{\xi}=\bigcup\left\{M_{\eta}: \eta \leq \xi\right\}, \xi<\omega_{1}$. Then $N_{\xi} \in \mathscr{B}_{\xi}$ and $\widetilde{Q}\left(N_{\xi}\right)=0$ and

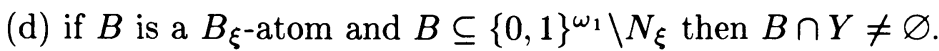

Let $(X, \mathscr{A})$ be a measurable space and $R$ a measure on $(X \times Y, \mathscr{A} \otimes \mathscr{C})$ with marginal $Q$ on $Y$. Note that $\mathscr{A} \otimes \mathscr{C}=(\mathscr{A} \otimes \mathscr{B}) \cap(X \times Y)$. Let $R^{\prime}$ denote the extension of $R$ to $\mathscr{A} \otimes \mathscr{B}$. By Lemma 1 , there exist measures $T_{x}^{\prime}, x \in X$, defined on $\mathscr{B}$ and satisfying the assertions of the lemma.

$$
\text { Let } L_{\xi}=\left\{x \in X: T_{x}^{\prime}\left(N_{\xi}\right) \neq 0\right\}, \quad \xi<\omega_{1} .
$$

Then $L_{\xi} \in \overline{\mathscr{A}}$, the completion of $\mathscr{A}$ w.r.t. $P$, the marginal of $R^{\prime}$ on $X$. Since $\widetilde{Q}\left(N_{\xi}\right)=0$ and since the marginal of $R^{\prime}$ on $\{0,1\}^{\omega_{1}}$ is $\widetilde{Q}$, we have $R^{\prime}\left(X \times N_{\xi}\right)=0$. It now follows from (b) of the lemma, that $P\left(L_{\xi}\right)=0$. Moreover $L_{\xi} \subseteq L_{\eta}$, $\xi<\eta<\omega_{1}$.

For each $x \in X$, define

$$
\begin{aligned}
r(x) & =\text { least } \xi \text { such that } x \in L_{\xi} \quad \text { if such a } \xi \text { exists, } \\
& =\omega_{1}, \quad \text { otherwise. }
\end{aligned}
$$

Let $\mathscr{F}_{x}=\bigcup\left\{\mathscr{B}_{\xi}, \xi<r(x)\right\}$. Clearly $\mathscr{F}_{x}$ is a field of sets and is in fact a $\sigma$-field if $r(x)$ is a successor ordinal or $\omega_{1}$. Observe now that

(e) $x \notin L_{\xi}, B \in \mathscr{B}_{\xi}$ and $B \cap Y=\varnothing$ imply $T_{x}^{\prime}(B)=0$.

To see this, note that by virtue of $(\mathrm{d}), B \subseteq N_{\xi}$ and since $x \notin L_{\xi}, T_{x}^{\prime}(B)=0$.

It immediately follows from (e) that $Y$ is $\left(T_{x}^{\prime}, \mathscr{F}_{x}\right)$-thick. Let $T_{x}^{*}$ be the restriction of $T_{x}^{\prime} \mid \mathscr{F}_{x}$ to $\mathscr{F}_{x} \cap Y$. Clearly $T_{x}^{*}$ is a charge on $\mathscr{F}_{x} \cap Y$, which is a sub-field of $\mathscr{C}$. For each $x \in X$, extend $T_{x}^{*}$ on $\mathscr{F}_{x} \cap Y$ to a charge $T_{x}$ on $\mathscr{C}$. We shall now verify that $\left\{T_{x}\right\}$ is a factorization of $Q$.

For $E \in \mathscr{A} \otimes \mathscr{C}$, choose $E^{\prime} \in \mathscr{A} \otimes \mathscr{B}$ such that $E=E^{\prime} \cap(X \times Y)$. Fix $\xi<\omega_{1}$ such that $E^{\prime} \in \mathscr{A} \otimes \mathscr{B}_{\xi}$. Then $E_{x}^{\prime} \in \mathscr{B}_{\xi}$ for each $x \in X$. Consequently, if $x \notin L_{\xi}$, 
$r(x)>\xi$, hence $E_{x}^{\prime} \in \mathscr{F}_{x}$, i.e. $E_{x}=E_{x}^{\prime} \cap Y \in \mathscr{F}_{x} \cap Y$. So $T_{x}\left(E_{x}\right)=T_{x}^{\prime}\left(E_{x}^{\prime}\right)$ for $x \notin L_{\xi}$. Therefore the map $x \rightarrow T_{x}\left(E_{x}\right)$ is $\overline{\mathscr{A}}$-measurable. Finally,

$$
\begin{aligned}
R(E) & =R\left(E^{\prime} \cap(X \times Y)\right)=R^{\prime}\left(E^{\prime}\right) \\
& =\int T_{x}^{\prime}\left(E_{x}^{\prime}\right) d P(x)=\int T_{x}\left(E_{x}\right) d P(x) .
\end{aligned}
$$

For the last part of the theorem, suppose that $\mathscr{D}$ is a countably generated sub$\sigma$-field of $\mathscr{C}$. Then we can find $\xi<\omega_{1}$ such that $\mathscr{D} \subseteq \mathscr{B}_{\xi} \cap Y$. Now if $x \notin L_{\xi}$, $\mathscr{D} \subseteq \mathscr{F}_{x} \cap Y$ and so $T_{x}=T_{x}^{*}$ on $\mathscr{D}$, and since $\mathscr{D}$ is a $\sigma$-field, $T_{x}^{*}$ is a measure on $\mathscr{D}$. (In fact, $T_{x}^{*}$ is countably additive on any $\sigma$-field contained in $\mathscr{F}_{x} \cap Y$ ).

REMARK. If $\mu$ is measure on a field $\mathscr{F}$ of subsets of a set $X, \mathscr{G}$ is a sub- $\sigma$-field of $\mathscr{F}$ and $A$ is $(\mu, \mathscr{G})$-thick, then it is routine to verify that the restriction $\nu$ of $\mu \mid \mathscr{G}$ to $\mathscr{G} \cap A$ is a measure. However, if $\mathscr{G}$ were only a sub-field, $\nu$ need only be a charge. Thus in the proof of Theorem 2 , it is possible that there exists $x \in X$ such that $T_{x}^{*}$ is a charge but not a measure on $\mathscr{F}_{x} \cap Y$. We mention in this connection the following example, often referred to as the de Finetti coin.

EXAMPLE. Let $X=\{0,1\}^{N}$ where $N$ is the set of positive integers, $\mathscr{F}=$ the field of clopen sets of $X$, when $X$ is equipped with the product of discrete topologies and $\mu$ the fair coin tossing measure on $\mathscr{F}$. Let $A$ denote the set of all sequences $\left(\omega_{1}, \ldots, \omega_{n}, \ldots\right)$ in $X$ for which $\omega_{i}=0$ for all sufficiently large $i$. Then it is easy to check that $A$ is $(\mu, \mathscr{F})$-thick and $\mu$ restricted to $\mathscr{F} \cap A$ is a charge but not a measure.

Combining Theorems 1 and 2, we get new necessary and sufficient conditions for a measure on a $\aleph_{1}$-generated $\sigma$-field to be perfect.

THEOREM 3. Let $(Y, \mathscr{C}, Q)$ be a measure space with $\mathscr{C}$ a $\aleph_{1}$-generated $\sigma$-field.

Then the following are equivalent:

(a) $Q$ is a perfect measure.

(b) For any measurable space $(X, \mathscr{A})$ and any measure $R$ on $A \otimes \mathscr{C}$ with marginal $Q$ on $Y$, there is a factorization of $R$.

(c) For any measurable space $(X, \mathscr{A})$, with $\mathscr{A}$ countably generated, and any measure $R$ on $\mathscr{A} \otimes \mathscr{C}$ with marginal $Q$ on $Y$, there is a factorization of $R$.

(d) For any measure $R$ on $\mathscr{B}^{1} \otimes \mathscr{C}$ with marginal $Q$ on $Y$, there is a factorization of $R$. to

Moreover if $\mathscr{C}$ is countably generated, each of the above conditions is equivalent

$\left(\mathrm{b}^{\prime}\right)$ For any measurable space $(X, \mathscr{A})$ and any measure $R$ on $A \otimes \mathscr{C}$ with marginal $Q$ on $Y$, there is a countably additive factorization of $R$.

The equivalence of perfectness and $\left(b^{\prime}\right)$ is just what Adamski proved in [1].

4. Normal conditional distributions. We now show how Theorem 2 can be used to obtain results on existence of normal conditional distributions.

LEMMA 2. Suppose $(Y, \mathscr{C}, Q)$ is a perfect measure space, $\mathscr{C}$ is $\aleph_{1}$-generated and $\mathscr{D}$ is a countably generated sub- $\sigma$-field of $\mathscr{C}$. Then there is a normal conditional distribution given $\mathscr{D}$.

Proof. In Theorem 2, take $X=Y, \mathscr{A}=\mathscr{D}$ and $R(E)=\int I_{E^{y}}(y) d Q(y)$, $E \in \mathscr{A} \otimes \mathscr{C}$. Let $\left\{T_{x}\right\}$ be a factorization of $R$ such that, for some set $N \in \mathscr{A}$ 
with $P(N)=0, T_{x}$ is a measure on $\mathscr{D}$ whenever $x \notin N$. Let $\mathscr{E}$ be a countable field which generates $\mathscr{D}$. Using routine measure-theoretic arguments, one can find $M \in \mathscr{D}$ with $Q(M)=0$ and such that for $x \notin M$, we have

(i) for each $E \in \mathscr{E}$, the map $x \rightarrow T_{x}(E)$ is $\mathscr{D}$-measurable on $X \backslash M$,

(ii) for each $E \in \mathscr{E}, T_{x}(E)=I_{E}(x)$

(iii) $T_{x}$ is countably additive on $\mathscr{D}$.

Condition (iii) now implies that (i) and (ii) hold for all $E \in \mathscr{D}$.

Next, for each $\mathscr{D}$-atom $D \subseteq M$, choose a charge $\nu_{D}$ on $\mathscr{C}$ such that $\nu_{D}(D)=1$. We can now define $\mu$ as follows:

$$
\begin{aligned}
\mu(y, C) & =T_{y}(C) \quad \text { if } y \notin M, \\
& =\nu_{D(y)}(C) \quad \text { if } y \in M .
\end{aligned}
$$

It is now straightforward to verify that $\mu$ is a normal conditional distribution given $\mathscr{D}$.

As in Dubins and Heath [8], say that a $\sigma$-field $\mathscr{D}$ is tail-like if there exist countably generated $\sigma$-fields $\mathscr{D}_{n}$ such that $\mathscr{D}_{n} \supseteq \mathscr{D}_{n+1}, n \geq 1$ and $\mathscr{D}=\bigcap_{n=1}^{\infty} \mathscr{D}_{n}$. As observed in [8], for such a $\mathscr{D}, D(y)=\bigcup_{n>1} D_{n}(y)$, where $D_{n}(y)$ is the $\mathscr{D}_{n}$-atom containing $y$. Since countably generated $\sigma$-fields contain their atoms, it follows that $D(y) \in \mathscr{D}$.

Using the previous lemma and a technique in [8], we can generalize Theorem 1 of Dubins and Heath [8].

THEOREM 4. Suppose $(Y, \mathscr{C}, Q)$ is a perfect measure space, $\mathscr{C}$ is $\aleph_{1}$-generated and $\mathscr{D}$ is a tail-like sub- $\sigma$-field of $\mathscr{C}$. Then there is a normal conditional distribution given $\mathscr{D}$.

PROOF. Let $\mathscr{D}=\bigcap_{n \geq 1} \mathscr{D}_{n}$, where each $\mathscr{D}_{n}$ is a countably generated sub- $\sigma$ field of $\mathscr{C}$ and $\mathscr{D}_{n} \supseteq \mathscr{D}_{n+1}, n \geq 1$. By Lemma 2 , for each $n \geq 1$, there is a normal conditional distribution $\mu_{n}$ given $\mathscr{D}_{n}$. By the Reverse Martingale Convergence Theorem, for each $C \in \mathscr{C}, \mu_{n}(y, C)$ converges $Q$-a.s. to $E\left(I_{C} \mid \mathscr{D}\right)$, the conditional expectation of the characteristic function of $C$ given the $\sigma$-field $\mathscr{D}$. Fix a Banach limit $l$ and define $\mu$ by $\mu(y, C)=l\left(\left\{\mu_{n}(y, C)\right\}\right), y \in Y, C \in \mathscr{C}$.

Since $\mu_{n}$ is a normal conditional distribution given $\mathscr{D}_{n}$, it is easily verified that $\mu$ is a normal conditional distribution given $\mathscr{D}$.

The above technique can be used in some special cases to show the existence of normal conditional distributions with additional desirable properties. We illustrate this with two examples.

THEOREM 5. Let $(Y, \mathscr{C}, Q)$ be a measure space and let $T$ be a measurable, measure-preserving transformation on $Y$. Let $\mathscr{I}$ denote the $\sigma$-field of invariant sets (i.e. $\mathscr{I}=\left\{E \in \mathscr{C}: T^{-1}(E)=E\right\}$ ). Then there is a normal conditional distribution $\mu$ given $\mathscr{I}$ such that $\mu(y, \cdot)$ is invariant and ergodic for each $y \in Y$. (i.e. $\mu(y, E)=\mu\left(y, T^{-1}(E)\right)$ for all $E \in \mathscr{C}$ and $\mu(y, A)=0$ or 1 for all $A \in \mathscr{F}$ ).

ProOF. Fix a Banach limit $l$ and for each $A \in \mathscr{C}$, and $y \in Y$, define $\mu(y, A)=$ $l\left(\left\{1 / n \sum_{i=0}^{n-1} I_{A}\left(T^{i} y\right)\right\}\right)$. To see that $\mu$ is a normal conditional distribution given $\mathscr{I}$, note that (a) by the Individual Ergodic Theorem, $\mu(y, A)=E\left(I_{A} \mid \mathscr{I}\right)$ a.s. $[Q]$, and (b) if $y, y^{\prime}$ belong to the same $\mathscr{S}$-atom, then $\mu(y, \cdot)=\mu\left(y^{\prime}, \cdot\right)$. It follows 
from the definition of $\mu$ that each $\mu(y, \cdot)$ is invariant and from condition (iv) in the definition of normal conditional distribution that each $\mu(y, \cdot)$ is ergodic.

Consider the special case when $Y$ is the unit circle, $\mathscr{C}$ is the Borel sets, $Q$ is the normalized Lebesgue measure and $T$ any nonperiodic rotation. Then Theorem 5 provides an affirmative answer to a question of Dubins and Heath in [8].

For the next example, let $(X, \mathscr{A})$ be a measurable space, $Y=X^{N}$ and $\mathscr{C}=\mathscr{A}^{N}$, where $N$ is the set of positive integers. For each $n \geq 1$, let $S_{n}$ denote the group of all permutations on $\{1, \ldots, n\}$. Every $\pi \in S_{n}$ induces a measurable bijection of $Y$, denoted by $\pi$ again, defined by $\left(x_{1}, \ldots, x_{n}, x_{n+1}, \ldots\right) \rightarrow\left(x_{\pi(1)}, \ldots, x_{\pi(n)}, x_{n+1}, \ldots\right)$. We say that a charge $\nu$ on $(Y, \mathscr{C})$ is symmetric if $\nu(E)=\nu(\pi E)$ for all $\pi \in \bigcup_{n \geq 1} S_{n}$.

Say that a symmetric charge $\nu$ on $\mathscr{C}$ is a power charge if

$$
\nu\left(A_{1} \times \cdots \times A_{n} \times X \times X \times \cdots\right)=\prod_{i=1}^{n} \nu\left(A_{i} \times X \times X \times \cdots\right)
$$

for all $n \geq 1$ and $A_{1}, \ldots, A_{n}$ in $\mathscr{A}$.

Let $\mathscr{S}$ denote the sub- $\sigma$-field of all symmetric sets, i.e. $\mathscr{S}=\{E \in \mathscr{C}: \pi(E)=E$ for all $\left.\pi \in \cup_{n \geq 1} S_{n}\right\}$.

THEOREM 6. Let $Q$ be a symmetric measure on $(Y, \mathscr{C})$. Then there is a normal conditional distribution $\mu$ given $\mathscr{S}$ such that for each $y \in Y, \mu(y, \cdot)$ is a power charge.

Proof. For each $y \in Y$ and $C \in \mathscr{C}$, define

$$
\mu_{n}(y, C)=\frac{1}{n !} \sum_{\pi \in S_{n}} I_{C}(\pi(y))
$$

Fix a Banach limit $l$ and define $\mu$ by

$$
\mu(y, C)=l\left(\left\{\mu_{n}(y, C)\right\}\right), \quad y \in Y, C \in \mathscr{C} .
$$

Once again using the Reverse Martingle convergence and straightforward calculations (as in Chapter $8, \S 1$ of [12]), it can be verified that $\mu$ satisfies all the asserted properties.

REMARK. Assume that singletons are measurable in $\mathscr{A}$. For $y \in Y$ such that $S(y)$, the $\mathscr{S}$-atom containing $y$, is infinite, (this happens iff $y$ is not a constant sequence), since $\mu(y, \cdot)$ is symmetric and since $\mu(y, S(y))=1$, it follows that $\mu(y, \cdot)$ is a charge and not a measure. Also the definition of $\mu$ is independent of $Q$. Hence, in view of condition ( $\mathrm{v}$ ) in the definition of normal conditional distribution, every symmetric measure is an average of power charges. This is similar in spirit to, but different from de Finetti's theorem. Also if $X=N$ and $\mathscr{A}$ the power set, for $y \in Y$ such that $S(y)$ is infinite, $\mu(y, \cdot)$ is not an independent strategic measure as defined in Purves and Sudderth [15]. To see this, first note that if $\gamma$ is a nondegenerate charge on $N$, and $\gamma^{\infty}$ is the independent strategic measure $\gamma \times \gamma \times \cdots$, then, for every $y^{\prime} \in N^{N}, \gamma^{\infty}\left(\left\{y^{\prime}\right\}\right)=0$ (see Lemma $2, \S 7$ of $[\mathbf{1 5}]$ ). Now using Lemma $2, \S 4$ of [15], it follows that $\gamma^{\infty}(S(y))=0$. However $\mu(y, S(y))=1$. 


\section{REFERENCES}

1. W. Adamski, Factorization of measures and perfection, Proc. Amer. Math. Soc. 97 (1986), 30-32.

2. D. Blackwell and L. E. Dubins, On existence and non-existence of proper, regular conditional distributions, Ann. Probab. 3 (1975), 741-752.

3. D. Blackwell and A. Maitra, Factorization of probability measures and absolutely measurable sets, Proc. Amer. Math. Soc. 92 (1984), 251-254.

4. D. Blackwell and C. Ryll-Nardzewski, Non-existence of everywhere proper conditional distributions, Ann. Math. Stat. 34 (1963), 223-225.

5. L. E. Dubins, On conditional distributions for stochastic processes, Proc. Sympos. Probability Theory, Univ. of Aarhus, Denmark, 1971.

6. —— Finitely additive conditional probabilities, conglomerability and disintegrations, Ann. Probab. 3 (1975), 89-99.

7. __ Measurable, tail disintegrations of the Haar integral are purely finitely additive, Proc. Amer. Math. Soc. 62 (1977), 34-36.

8. L. E. Dubins and D. Heath, With respect to tail sigma-fields, standard measures possess measurable disintegrations, Proc. Amer. Math. Soc. 88 (1983), 416-418.

9. D. Heath and W. D. Sudderth, On finitely additive priors, coherence and extended admissibility, Ann. Stat. 6 (1978), 333-345.

10. D. A. Lane and W. D. Sudderth, Diffuse models for sampling and predictive inference, Ann. Stat. 6 (1978), 1318-1336.

11. E. Marczewski, On compact measures, Fund. Math. 40 (1953), 113-124.

12. P. A. Meyer, Probability and potentials, Blaisdell, Waltham, Mass., 1966.

13. K. Musial, Inheritness and perfectness of measures by thick subsets, Measure Theory, Oberwolfach 1975, Lecture Notes in Math., vol. 541, Springer-Verlag, Berlin and New York, 1976, pp. 31-42.

14. J. K. Pachl, Disintegration and compact measures, Math. Scand. 43 (1978), 157-168.

15. R. A. Purves and W. D. Sudderth, Some finitely additive probability, Ann. Probab. 4 (1976), 259-276.

16. C. Ryll-Nardzewski, On quasi-compact measures, Fund. Math. 40 (1953), 125-130.

17. V. V. Sazonov, On perfect measures, Amer. Math. Soc. Transl. (2) 48 (1965), 229-254.

18. R. Sikorski, Boolean algebras, 2nd edition, Springer-Verlag, Berlin and New York, 1964.

Stat.-Math. Division, The Indian Statistical Institute, Calcutta 700035, InDIA

Department of Mathematics and Computer Science, University of Miami, Coral Gables, Florida 33124 